Hermann H. Dieter | $\begin{aligned} & \text { Arbeitskreis Deutsch als Wissenschaftssprache e. V. (ADAWIS), } \\ & \text { hh.dieter@t-online.de }\end{aligned}$

\title{
Sprachenvielfalt, Erkenntnisfreiheit und Politik: Ein spannungsgeladenes Wechselspiel
}

\section{Sprachenvielfalt und Erkenntnis. Vielfältiges Denken contra einfältige Affirmation}

Ein kurzer Blick auf Wissenschaftstheorie und -geschichte zeigt, dass gerade die Vielfalt wissenschaftlichen Sprechens und sprachlich reflektierten Denkens, sowie ent $>$ sprechend differenzierter Weltsichten seit Beginn des $>$ Baconschen Zeitalters` (1620) Erkenntnispotenziale freigesetzt hat, die sich auf Lateinisch, also in der Sprache der damaligen $>$ Affirmationswissenschaft $<$, niemals hätten entwickeln können. ${ }^{1}$

Der aktuelle Hang, Drang, ja Zwang hin zu der >einzigen Wissenschaftssprache Englisch nährt deshalb die Befürchtung, die Wissenschaft insgesamt befände sich auf dem Weg in eine neue Scholastik, weg vom ergebnisoffenen wissenschaftlich Sprechen und sprachlich reflektierten Denken, hin zum Austausch und Platzieren von Lehrsätzen in der einzigen dafür affirmierten und sich dadurch selbst affirmierenden Sprache.
Wissenschaftliches

Arbeiten lässt sich nur international betreiben. Dazu bedarf es jedoch einer universellen Publikationsund Kongresssprache, deren Funktion zurzeit dem Englischen zukommt. Dessen hoher Wert für diesen Zweck ist kaum bestreitbar. Allerdings wird selbst dort, wo es nicht nur um die weltweite Verbreitung fertiger Erkenntnisse geht, sondern auch schon dort, wo solche erst gewonnen werden, bedenkenlos auf das Englische gesetzt. Es ist jedoch ein (Selbst-)Betrug des Wissenschaftsbetriebs, wenn die Sprache der internationalen Kommunikation als die universelle Wissenschaftssprache gehandhabt wird. Eine solche kann es nicht geben, sondern nur natürliche Sprachen, deren Lexik für Zwecke wissenschaftlichen Sprechens mehr oder weniger umfänglich ausgebaut wurde. 
Dank dieser sprachlich-affirmativen Rückkopplung können Naturwissenschaftler fast ungestört sich selbst und anderen gegenüber behaupten, der Gebrauch eines Einheitsidioms in den Natur- und Technikwissenschaften sei doch unproblematisch, denn die von ihnen gesuchten Wahrheiten seien grundsätzlich objektiv benennbar, wenn alle dieselbe Sprache benutzten.

Allerdings erübrige sich der Streit um die >richtige`Sprache in nicht allzu ferner Zukunft, denn beispielsweise in der biomedizinischen Forschung würden alltagssprachliche Erklärungen dank neuer und immer präziserer bildgebender Verfahren ohnehin allmählich überflüssig. Kultursprachliche Besonderheiten dürften bei der Wissensgenerierung allenfalls in den Geistes- und Kulturwissenschaften weiterhin noch eine gewisse Rolle spielen.

\section{Ein kleiner Versuch in Erkenntnistheorie}

\subsection{Vom Anschaulichen zum Unanschaulichen}

Jede innere, zunächst noch bildhafte Eingebung, jedes Bild eines natürlichen Phänomens und erst recht jedes technisch elaboriert erzeugte äußere Bild - etwa aus einem Messgerät - selbst jede mathematische Formel bedarf jedoch der sinnlichen Belebung und Deutung mit Mitteln der (unpräzisen) Alltags- oder Umgangssprache. Jedenfalls kann eine zunächst rein bildhafte Erkenntnis nur so aus der reinen Anschauung, dem Labor oder der eigenen biologisch-neuronalen Denkstube in die Außenwelt >über(ge)setzt « werden und mit ihr in Beziehung treten. ${ }^{2}$

Exaktes Beobachten schult sich sogar am besten beim Beschreiben des eigenen Blickwinkels auf äußere und innere Bilder - nicht nur für das eigene, sondern auch für das Denken anderer. Beispielsweise wird das botanische Phänomen mit dem deutschen Namen >Maiglöckchen`im Englischen als >Lily of the valley` und im Französischen als `Le muguet ‘ in Sprache übersetzt. Beide Sprachen sehen also von der Glöckchenform und -funktion seiner Blüten ab, ebenso wie das Deutsche vom Ort seines bevorzugten Auftretens und seinem Duft.

Beobachtung und Beschreibung `natürlich ‘ sichtbarer (Teil-)Phänomene und (Teil-)Gestalten sind allerdings nicht das eigentliche Ziel naturwissenschaftlicher Erkenntnisprozesse, sondern vielmehr die Enttarnung oder Freilegung der Kausalketten, über die erstere miteinander in Beziehung stehen (könnten). Deshalb strebt jeder Naturforscher danach, `seine` Be- 
obachtungen mit anderen durch eine verallgemeinernde $=$ abstrahierende Hypothese zu verbinden. Dieser Abstraktionsvorgang kann wiederum nur sprachlich strukturiert und bebildert sein. Nicht zu Unrecht wird von ihm jedenfalls erwartet, dass er weitere, bisher anscheinend nicht beobachtbar gewesene Tatsachen voraus `sagt $\iota$, solche also nicht nur - wie man das gerne so vielen sogenannten Wetterprognosen anlasten würde - willkürlich vorausbehauptet.

Die Richtigkeitsvermutung für die abstrahierende Hypothese gilt aber nur solange, wie sie empirisch/experimentell nicht widerlegt wurde. Zur wissenschaftlichen Fairness gehört es deshalb, nicht willkürlich irgendwelche bisher unbekannte Tatsachen zu behaupten, sondern nur solche, deren Existenz erfahrungsbasiert (wissenschaftsmethodisch) entweder vollständig oder partiell widerlegbar $=$ falsifizierbar wäre, falls sie nur erfunden wären. Im Ergebnis dieser Überprüfung wird die Anfangshypothese dann entweder verworfen, modifiziert, erweitert oder bestätigt.

\section{2. Über Unanschauliches lässt sich sprachvielfältig streiten}

Über die >objektive`s sprachliche Ausformung der unumgänglichen Übersetzung kultursprachlich gefasster Beobachtungen in experimentell falsifizierbare Hypothesen lässt sich je nach Kulturkreis in eben der Sprache, die diesen reflektiert, hoffentlich auch in Zukunft weiterhin erkenntnisfördernd streiten. Die sprachliche Präzisierung falsifizierbarer Hypothesen und Theorien kann aber nur im Selbstgespräch oder in größeren Gesprächsrunden gelingen und auch nur in einer Fachsprache, die auf einer allen Gesprächspartnern gemeinsamen und möglichst gut beherrschten Gemein- oder natürlichen Sprache aufsetzt. ${ }^{3}$

Im vorliegenden Beispiel ist das deutschsprachige Bild des (blühenden) >Glöckchens` Ausgangspunkt für eine erste Hypothese darüber, was die >unanschaubare`Funktion dieser Blütengestalt mit der anschaulichen Metapher, die ihr sprachlich intuitiv zugesprochen wurde, zu tun haben könnte. Tatsächlich sind Sprachbilder oder Metaphern, die ihnen zugrunde liegende Daten sichtbar machen sollen, monoperspektivische Artefakte mit nur vorläufigem, grundsätzlich begrenztem Erklärungswert. Dennoch sind sie ein intuitiv gesetzter und brauchbarer Ausgangspunkt für den Weg zu neuen Erkenntnissen. ${ }^{4}$ Ihre widerspruchsfreie Erweiterung oder wörtliche bis

3 Thielmann: Deutsche und englische Wissenschaftssprache im Vergleich.

4 Drewer: Die kognitive Metapher, dort insbesondere die Kapitel 2-4 mit weiterführenden Hinweisen auf Radman (1976), Lakoff/Johnson (1980, 1987) und Sweetser (1990). 
begriffliche Umdeutung für einen neuen Zielbereich der Erkenntnis muss und wird von Sprache zu Sprache jedoch sehr unterschiedlich verlaufen.

Die Kognitive Linguistik hat uns Einblicke in neuronale Prozesse ermöglicht, die abbilden, wie unterschiedlich die sprachenverschiedene Wahrnehmung aussehen kann. ${ }^{5}$ Die Hypothese dazu ist schon wesentlich älter. Genau darauf spielt Werner Heisenberg mit seinem Hinweis an, demzufolge Wissenschaft im Gespräch entstehe. ${ }^{6}$ Dies gilt nicht nur für die einstigen, nur umgangssprachlich diskutierbar gewesenen Streitpunkte etwa der Atomphysik, ${ }^{7}$ sondern genauso z.B. für Streit und Verständigung darüber, wie und warum die hier aus nur drei sprachlichen Blickwinkeln präzisierten Eigenschaften einer botanischen Erscheinung evolutionär exakt in der (Teil-)gestalt einer Glöckchenreihe kulminieren konnten. Dennoch wird das spezifische Erkenntnispotenzial jeder kulturell eingebundenen Umgangssprache namentlich von Naturwissenschaftlern heftig bestritten.

Fazit: Um die Wirklichkeit zu strukturieren und zu verstehen, erdenken wir zunehmend widerspruchsfreie Theorien, deren Abfolge uns der Wirklichkeit asymptotisch näherbringt. Eine neue Hypothese ist uns zunächst zwar nur geistig präsent. Doch praktisch jede lässt sich dadurch veranschaulichen, dass wir ihren empirischen Wahrheitsgehalt an Tatsachen und vor empirisch gesicherten Bildern prüfen, die uns sinnlich bereits zugänglich sind. In anderen Worten: Um den noch hypothetischen Zielbereich der Erkenntnis perspektivisch `auszuleuchten`, nutzen wir bei dieser $>$ mesokosmischen Transformation ${ }^{8}$ intuitiv Metaphern, die von bereits ausgeleuchteten Tatsachen und Befunden rausgehen $<$. Eine solche Metapher war oben im Text das Bild des `Glöckchens`, obwohl von vornherein klar war, dass eine `Glöckchenreihe`, die (warum eigentlich?) als Blüte gut funktioniert, weder aus Metall bestehen noch - zumindest für menschliche Ohren - hörbar >läuten k kann.

Ganz ähnlich verhalf die Metapher vom `Schwarzen Loch`den Kosmologen bei der Generierung von Hypothesen über die wahre Gestalt, Funktion und Geschichte eines bis dato unbekannten Phänomens im Universum. ${ }^{9}$ Irgendwann wird eine präzisere Metapher an seine Stelle treten, genauso wie jemand, der schon einmal eine >crème renversée` genießen durfte, diese nicht weiterhin als `Pudding < bezeichnen sollte, obwohl sie (s.u.) auf den ersten Blick so auszusehen scheint. 


\subsection{Jeder Mensch kommt sprachlich als Wissenschaftler 'zur Welt`}

Einen runden Gegenstand benennt ein Kleinkind zunächst immer wie den zuerst `begriffenen $`$ und als >Ball in Sprache übersetzten Gegenstand. Erst durch Erfahrung und Widerspruch lernt es, dass >runde` Dinge in unterschiedlichsten Gestaltvarianten vorkommen und ent $>$ sprechend unterschiedlicher Benennungen bedürfen.

Eine bestimmte Süßspeise französischer Herkunft, die zunächst als Pudding imponiert, ist zwar keiner, wird aber erfahrungsgemäß von muttersprachlich deutschsprachigen Kleinkindern (und Erwachsenen!) zunächst doch `so genannt . Erst durch Vergleich und Widerspruch wird sie sich allmählich als davon unterschiedlich bebildern, nämlich als `crème renversée`. Ihr Name verweist statt auf die einst Blutwurst-ähnliche Konsistenz ${ }^{10}$ eines >Puddings` auf das (abgeschlossene) Bild ihrer physischen Herauslösung aus der Kochform.

Das neue Bild schließt diesen Erkenntnisprozess ab und zeigt, dass wir das bisher Unbekannte `begriffen` haben. Ganz ähnlich diente das Bild des `Glöckchens` oder der `Glöckchenreihe` als Ausgangspunkt für eine Hypothese darüber, was die >unanschaubare` Funktion dieser Blüte mit ihrem anschaulichen Bild zu tun haben könnte. Als ähnlich erkenntnisleitendes Bild dient in meinem Fach etwa das Bild der >Anstandsdame`, engl. >chaperone . Es steht in der Biochemie für eine in den 1990er-Jahren entdeckte Gruppe von Proteinfaltungs-Hilfsproteinen. ${ }^{11}$ Deren anglophone Entdecker nahmen es dafür, trotz seines in der Alltagssprache völlig anderen Kontextes, sozusagen sanstandslos` in Beschlag.

Wissenschaftliches Sprechen ist also eine fachtypische Art des Sprechens, die dennoch unlösbar mit einer Gemein- oder natürlichen Sprache verbunden ist. ${ }^{12}$ Eine $\gg$ Wissenschaftssprache , die nicht auf eine solche Sprache und insbesondere ihre Metaphern aufsetzt, wäre vielleicht konstruierbar, für Nicht-Fachleute jedoch inhaltsleer. Der Philosoph und Physiker C. F. v. Weizsäcker schrieb: »Die so genannte exakte Wissenschaft kann niemals und unter keinen Umständen der Anknüpfung an das, was man die natürliche Sprache oder die Umgangssprache nennt, entbehren. Es handelt sich stets nur um einen Prozess der vielleicht sehr weit getriebenen Umgestaltung derjenigen Sprache, die wir immer schon sprechen und verstehen. $\ll^{13}$

10 Blutwurst $=$ black boudin (frz.), als $>$ pudding $\prec=$ Süßspeise seit dem 16. Jh. im Englischen und von dort ins Deutsche (Kluge: Ethymologisches Wörterbuch).

11 Belegt z.B. in Saibil: Chaperone machines.

12 Thielmann: Deutsche und englische Wissenschaftssprache im Vergleich.

13 Weizsäcker: Die Einheit der Natur, S. 65. 
Fazit: Schon jeder Säugling ist, jedenfalls sobald er beginnt, die Welt muttersprachlich zu begreifen und zu strukturieren, ein neuer und tatsächlich >geborener`Wissenschaftler. Nicht von ungefähr wurde Albert Einstein bis zu seinem Lebensende die Neugier und Erkenntnisoffenheit eines Säuglings zugesprochen.

\subsection{Konstruktivistischer vs. objektivistischer Erkenntnisansatz}

Der soeben skizzierte sprachlich-intersubjektive Erkenntnisansatz heißt >konstruktivistisch ${ }^{14}$ Er rechnet nicht damit, dass sich die Wirklichkeit jemals >objektiv` versprachlichen ließe, sondern hält aus sprachlicher Sicht nur iterativ-diskursiv verfasste Annäherungen möglichst vieler Subjekte an eine oder die objektive Wirklichkeit für wahrheitsverträglich. In ihm ist der Mensch (das Subjekt) »kein passiver Beobachter einer außersprachlichen Wirklichkeit, sondern ein aktiver, handelnder Teilnehmer «, der sie nur "partizipierend " (aber eben deshalb auch immer nur partiell) zu erkennen vermag. ${ }^{15}$ Ein sozusagen endgültiges, dazu noch grammatikalisch und lexikalisch stark verkürztes Einheitsidiom reicht deshalb auch in der Naturwissenschaft nicht aus, um den Blick auf die Wirklichkeit immer wieder zu präzisieren und neu auszurichten, statt ihn nur zu festigen.

Je mehr Sprecher wissenschaftstauglicher Muttersprachen um einen gemeinsamen Gegenstand, eine gemeinsame Beobachtung streiten, desto deutlicher und multiperspektivischer werden die resultierenden Erkenntnisse ausfallen. Kaum ein wissenschaftliches Gespräch dürfte erhellender sein als der Vergleich muttersprachlich unterschiedlicher Termini und Metaphern für als verwandt erachtete Gedanken oder empirische Sachverhalte. Die immer weitere Präzisierung des Denkens dank muttersprachlicher Intuition ist auch die Voraussetzung für die Freiheit der Erkenntnis im inneren Selbstgespräch und die freie Rede nach außen. Diese Freiheit kann gerade den naturwissenschaftlich Forschenden nicht irgendeine Staatsverfassung garantieren, sondern nur ein mehrsprachig verfasster und ein sprachlich auch von innen entsprechend gepflegter Wissenschaftsbetrieb.

Fazit: Auch in den Naturwissenschaften hat Sprache nicht nur die extern-kommunikative Funktion zur Belebung des so heiß begehrten >internationalen Austauschs $\triangleleft{ }^{16}$ Viel stärker noch hat sie eine intern-kommunikative und -kognitive Funktion: beim persönlichen (inneren) Ringen

15 Ebd., S. 35.

16 Trabant: Globalesisch für europäische Mehrsprachigkeit. 
um Erkenntnis, im ergebnisoffenen inter-nationalen Gespräch mit Fachkollegen, beim Übertragen von Erkenntnissen von der intuitiven eigenen in eine andere, später erlernte Sprache.

Vorläufige oder scheinbar fertige Erkenntnisse dagegen lassen sich mehr oder weniger präzise in fast jeder beliebigen Sprache darstellen. Die Übersetzung des muttersprachlich Formulierten dorthin präzisiert dieses dann sogar weiter, wie alle bestätigen werden, die dies zumindest schon einmal beim Übersetzen eigener Texte etwa ins Englische erleben durften.

Im Idealfall sollte also auch jeder Naturwissenschaftler seine Denk- und Muttersprache mindestens so gut beherrschen wie sein Wissenschaftsenglisch. Eine Bildungspolitik und ein Wissenschaftsbetrieb, die zulassen, dass wir weiterhin nur noch das Englische zu Lasten aller anderen (noch) wissenschaftstauglichen Sprachen ausbauen, vergehen sich an der Freiheit des Denkens und der Erkenntnis.

\section{5. `English only«: Kommt ein überwunden geglaubtes Problem zurück?}

Wir erleben zurzeit, wie sich Forschung und Lehre weltweit auf immer weniger und bald womöglich nur noch eine einzige Sprache zurückziehen. Erneut besteht die Gefahr, dass diese Sprache - ähnlich wie das Gelehrtenlatein bis zum ausgehenden Mittelalter - zu einem formelhaften, nur rhetorisch befragbaren Idiom erstarrt, in dem sich seinerzeit anerkanntes (Buch-)Wissen immer nur neu kompilieren und affirmieren ließ, Neues jedoch - mangels nicht-formelhafter Sprachen - nicht denkbar war. ${ }^{17}$

Droht uns wieder die Verordnung des scheinbar einzig richtigen Wissens? Also etwa darüber, wer die Existenz des Menschen zu deuten habe und wohin oder wie sein Zusammenleben mit seinesgleichen und der natürlichen Umwelt zu lenken sei? Könnte es sein, dass es falsch ist, jeden naturwissenschaftlich neu ermittelten Sachverhalt mittels einer ökonomisch gelenkten Sprache ${ }^{18}$ sofort auf seine ökonomische Profitabilität zu prüfen d.h. lange bevor Antworten darauf gesucht und gefunden wurden, welche Art des Zusammenlebens in Zukunft gelten soll - und dazu wiederum nur rhetorische Fragen zuzulassen?

Schon der mittelalterliche Wahrheitsanspruch war jedenfalls an die Behauptung der >Wissenden ' gebunden, die Wirklichkeit und verbindliche Erkenntnisse zur Art des Zusammenlebens der Menschen ließen sich ১objektiv richtig`nur auf eine einzige Weise versprachlichen. Andere Spra- 
chen als das Lateinische waren als (scheinbar) objektivierendes Werkzeug der Erkenntnis nicht anerkannt und zur Wahrheitsführung infolgedessen weder fähig noch berechtigt. Als dann der neue konstruktivistische, hypothesen- und theoriegeleitete Erkenntnisprozess in den Mittelpunkt rückte, musste man auf die Volkssprachen und deren jeweils eigenen sprachlichen Blickwinkel zurückgreifen, die das lateinische Einheitsidiom rasch als überflüssig erscheinen ließen und entbehrlich machten. ${ }^{19}$

\section{Treiber der modernen Sprachverengung}

\subsection{Internationalisierung $=$ Anglophonisierung der akademischen Lehre?}

Wissenschaft wäre keine ohne die Entdeckung des Nichtwissens vor rund 400 Jahren und den aus Neugier erwachsenen Wunsch, es durch stete Produktion von Wissen aufzulösen und widerlegtes Wissen konsequent zu verwerfen. Bisher unwiderlegt ist allerdings die Erkenntnis, dass jedwede Erkenntnis diskursiv, d.h. sprachgebunden zustandekommt und jede Sprache ihre eigenen Erkenntniswege besitzt. Nur fertige Erkenntnisse lassen sich zumindest in den Naturwissenschaften auch sprachunabhängig, d.h. in einer beliebigen, jedoch wissenschaftlich ausgebauten Sprache darstellen. Deshalb sind die internationale Vernetzung, das Streben nach steter Erweiterung des Wissens und die Fähigkeit, wissenschaftlich mehrsprachig zu kommunizieren unverzichtbare Merkmale jeder Art, Wissenschaft >ehrlich', d.h. erkenntnis- statt nutzenorientiert zu betreiben. Wissenschaftler dieser Art sind auch in besonderer Weise befähigt, den interkulturellen Austausch voranzubringen, denn die genannten Merkmale erziehen »zu Neugier, kultureller Offenheit und gegenseitiger Toleranz $\aleph^{20}$ im Denken und Handeln.

Diesem Befund und Wunsch trägt die Strategie der Wissenschaftsminister/innen von Bund und Ländern für die Internationalisierung der Hochschulen in Deutschland von 2013 durchaus Rechnung. Sie wäre, würde sie denn konsequent verfolgt, ein vielversprechender Weg, um den internationalen Austausch durch Fremdsprachen- und Deutschkurse zu fördern. Zu diesem Zweck müssten die Kurse allerdings eng und verpflichtend mit dem jeweiligen Fach verzahnt werden.

Um sinternationale`Studenten anzuwerben, setzen jedoch immer mehr Hochschule(n) auf Englisch als ausschließliche Lehrsprache. ${ }^{21}$ Verbindlich zu 
absolvierende fachbegleitende Deutschkurse sind nicht vorgesehen; all diese Kurse sind freiwillig, eine Erfolgskontrolle findet im Verlauf des Studiums nicht statt. Damit verzichten die Hochschulen auf ein verbindliches Merkmal von `Inter-Nationalität`, nämlich verbindlich mehrsprachige Lehre und Kommunikation. Sie blockieren dadurch den Erwerb sprachgebundener interkultureller Kompetenzen, die Integration der akademischen Absolventen aus dem Ausland in den inländischen Arbeitsmarkt und ihre gesellschaftliche Teilhabe. Auf die verfassungsrechtlichen Bedenken gegen ausschließlich englischsprachige Lehrangebote der Hochschulen gehe ich später ein.

Immer mehr Hochschullehrer beklagen dementsprechend zahlreiche Mängel der aktuellen Internationalisierungs- und Lehrpraxis der Hochschulen mit entsprechend negativen Folgen für die in- und ausländische Studentenschaft, so etwa:

- die oft absolut unzureichende Deutsch-, aber auch Englischkompetenz der ausländischen Studenten,

- den Verlust erworbener landessprachlicher Kenntnisse infolge Fixierung auf `English only<,

- fehlende Kenntnisse in der jeweiligen Fach- und Wissenschaftssprache. $^{22}$

Offenbar ist hier bereits ein Abwärtswettbewerb hinsichtlich der Sprachanforderungen - und damit hinsichtlich der Qualität der Lehre für alle Beteiligten - in Gang gekommen, denn im Wettbewerb um ausländische Studenten kann sich keine Universität einen Alleingang hin zu höheren Anforderungen leisten. Aus Angst, die Zielzahlen der ausländischen Studienanfänger nicht zu erreichen und deshalb Mittelkürzungen in Kauf nehmen zu müssen, meiden die Hochschulen auch eigene Zulassungsverfahren. Stattdessen werden, um sich der Problemfälle 'pragmatisch und schnell zu entledigen, Leistungspunkte (`credit points $`$ ) auch für zweifelhafte Leistungen vergeben.

Philipp Oswalt, Professor für Architekturtheorie an der Universität Kassel, und Marina Adams, Germanistin am deren Internationalem Studienzentrum, prangerten im März 2017 diese Missstände an. ${ }^{23}$ Ihre Beobachtungen nähren den Verdacht, dass das Projekt der Internationalisierung der öffentlichen Hochschulen Deutschlands zu einem Fetisch verkommen ist. Die Hochschulen missbrauchen es dazu, gegen entsprechende Landescomings, Studienerfolg und soziale Integration «. 
mittel wahllos die Zahl ihrer internationalen Studenten zu erhöhen. Die wirkliche Inter-Nationalisierung, nämlich die Förderung des interkulturellen Verständnisses oder die längerfristige Integration der ausländischen Absolventen, ja sogar die Qualität der Lehre, bleiben dabei auf der Strecke. Nicht einmal die Englischkenntnisse der begehrten `Internationalen « werden verbindlich und einheitlich überprüft.

Ausländische wie inländische Studenten sowie Dozenten leiden erheblich unter dieser Fehlentwicklung. Nach unserer Kenntnis gibt es in keinem deutschen Bundesland die ernsthafte Absicht, das Internationalisierungsgebaren ihrer Hochschulen in zweckdienliche Bahnen zu lenken. ${ }^{24}$

\subsection{Internationalisierung = Ökonomisierung der akademischen Ausbildung?}

Seit geraumer Zeit bemächtigt sich ein Wettbewerbs- und Effizienzdenken der Wissenschaft, das in der Wirtschaft möglicherweise zielführend ist, auf die Wissenschaft übertragen jedoch an deren Grundfesten rüttelt: Wissenschaftlich unhaltbare, jedoch wissenschaftlich daherkommende Maßzahlen sollen wissenschaftliche Leistungen scheinbar objektiv quantifizieren. ${ }^{25}$ Stattdessen provozieren sie schier endlose Ketten redundanter oder entbehrlicher Mini-Veröffentlichungen sowie bizarre Verzerrungen im gesamten Publikationswesen bis hin zur Wissenschaftsfälschung. ${ }^{26}$ Im Konkurrenzkampf um ein möglichst hohes Ranking helfen Eigenzitate und Zitationskartelle oft mehr als objektiv zitierenswerte Forschungsergebnisse. Von 100 schriftlich zitierten Publikationen werden 70 bis 90 anscheinend überhaupt nicht von Dritten gelesen. Verlage zwingen ihre Autoren, vorrangig das eigene Journal zu zitieren, um wissenschaftliche Reputation vorzutäuschen. ${ }^{27}$ Laborforschung und konventionelle Methoden, die kurzfristigen Erfolg versprechen, werden gegenüber langfristigen Studien bevorzugt. ${ }^{28}$

Anstatt sich inhaltlich zu verantworten, sind universitäre Forscher zunehmend gezwungen, durch marktschreierische Schlagzeilen aufzufallen, letztens beispielsweise an der Universität Heidelberg in Form der voreiligen Ankündigung eines wissenschaftlich invaliden Bluttests auf Brustkrebs. ${ }^{29}$ Um in einer wissenschaftlichen Rangliste nach oben zu rücken, wurden 
an anderer Stelle sogar schon Autorenschaften ${ }^{30}$ und renommierte Gastwissenschaftler ${ }^{31}$ teuer reingekauft . Zunehmend wird der Forschung ein Nützlichkeitsparadigma auferlegt, die Grundlagenforschung gerät unter Rechtfertigungszwang. Der Weg zur Fremdsteuerung durch kapitalkräftige Geldgeber ist da nicht mehr weit. ${ }^{32}$

Wissenschaftliche >Ausrufer oder $>$ Anpreiser , die sich im Wettbewerb um kapitalkräftige Geldgeber gegenseitig ausstechen oder überbieten, degradieren Lehre und Forschungsfelder zu marktkonformen Konsumgütern. Forschungsergebnisse und Bildung verlieren nach und nach ihr Existenzrecht `sui generis`, werden verhandelbar und zur jederzeit verfügbaren Handelsware. Unter dem Einfluss des erfolgsfixierten, affirmativen Paradigmas der Wirtschaft verkommt das Recht des wissenschaftlichen Arbeitens auf Irrtum zu einem »Skandalon «; (schon jetzt) »bringt dies die Arbeitsweise zumal der kognitiven Naturwissenschaft in eine unauflösbare Bedrängnis «, 33 denn infolge der exorbitanten Kosten ihrer Wissensproduktion sind deren Disziplinen auf wirtschaftliche Erfolge besonders heftig angewiesen.

Geisteswissenschaften dagegen, deren Erkenntnisse sich längst nicht so teuer und nutzbringend vermarkten lassen, befinden sich mit Ausnahme weniger Modefächer finanziell auf Entzug, während sie sich zum sprachlichen Rückzug auf Englisch oft schon deshalb zwingen lassen (oder dies selbst tun), um vorgeblich überhaupt noch gehört und gelesen zu werden. Dies ist desto bedenklicher, wie diesen Fächern angesichts des Wildwuchses technischer Machbarkeiten die wichtige Aufgabe zufällt, kulturwissenschaftlich basiertes, d.h. vor allem sprachgebundenes Bewertungs- und Orientierungswissen bereitzustellen und aktiv bekannt zu machen.

Fazit: Im Zusammenhang mit der Orientierung der Wissensproduktion und der akademischen Ausbildung auf einen globalen >Markt mag es ökonomisch erscheinen, der Forschung und Lehre eine Einheitssprache $\mathrm{zu}$ verordnen, die vorgeblich weltweit verstanden wird. Wissenschaft ist jedoch mehr als nur internationale Kommunikation. Eine reinzige Wissenschaftssprache für wissenschaftliches Sprechen und sprachlich reflektiertes Denken kann es nicht geben, allenfalls eine solche zur Beteuerung und Verbreitung naturwissenschaftlicher Erkenntnisse. Die Propagierung einer sich selbst affirmierenden (s.o., Kap. 1, zweiter Absatz) Einheitssprache mag für bestimmte, kommunikatorisch beschränkte Publikationsformate wohl 
hinnehmbar sein; eine notwendige intrinsische Voraussetzung wissenschaftlichen Arbeitens wäre jedoch ad absurdum geführt, wenn eine vollkommen alltagsfremde und formalisierte Sprache als >die Wissenschaftssprache allmählich alle anderen wissenschaftlich ausgebauten Sprachen aus diskursiven Erkenntnisprozessen und der akademischen Lehre verdrängen dürfte.

\section{Was ist zu tun?}

\subsection{Verfassungsrecht}

Das Oberste italienische Verwaltungsgericht stoppte 2018 die Narrenfreiheit italienischer Hochschulen beim Anbieten englischsprachiger Curricula. $^{34}$ Vorausgegangen war 2012 ein Beschluss der Mailänder Hochschule Politecnico di Milano, ab 2014 alle weiterführenden Studiengänge und Doktorandenprogramme nur noch auf Englisch anzubieten. Etwa 100 Hochschullehrer und Studenten hatten sich daraufhin durch die Instanzen geklagt und waren damit bis hinauf zur Ebene des Verfassungsgerichtshofs erfolgreich. Er befand am 21. Februar 2017, fremdsprachige Studienangebote seien zwar nicht zu beanstanden. Aus verfassungsrechtlicher Sicht dürften sie jedoch keinesfalls die gleichen Angebote in der Landessprache aus den Curricula verdrängen. Hierfür setzte er folgende Bedingungen:

1. Das Italienische genießt Vorrang vor anderen Sprachen, denn es ist offizielles Verständigungsmittel in allen öffentlichen Einrichtungen des Landes und Teil der Identität des italienischen Staates.

2. Studienbewerbern darf der Zugang zu den Hochschulen nicht verwehrt werden, wenn sie nur die Landessprache beherrschen.

3. Ein Zwang zu fremdsprachiger Lehre würde die Lehrfreiheit verletzen.

4. Komplette fremdsprachige Studiengänge sind mit der Verfassung vereinbar, wenn sie solchen in italienischer Sprache zur Seite gestellt sind.

5. Einzelne Lehrveranstaltungen, die aufgrund ihrer »Besonderheiten und Spezifika « ausschließlich fremdsprachig erteilt werden, sind zulässig. Sie müssen jedoch nach Art und Umfang sinnhaft, verhältnismäßig und angemessen sein und »den Vorrang der italienischen Sprache ebenso respektieren wie das Prinzip der Gleichheit, des Rechts auf Bildung und der Freiheit der Lehre«.

34 Der gesamte Ablauf dieses Rechtsstreites von 2012 bis 2018 mit allen relevanten Dokumenten und Erklärungen ist einzusehen auf der Internetseite des Arbeitskreises Deutsch als Wissenschaftssprache: <http://adawis.de/ausland/italien> (Zugriff: 7.11.2019). 
Der Verfassungsgerichtshof verwies die Causa deshalb an die Vorinstanz, den Consiglio di Stato, mit dem Auftrag zurück, in diesem Sinne endgültig zu entscheiden. Dieser gab den seit 2012 klagenden Studenten und Dozenten im Januar 2018 vollumfänglich Recht.

Studiengänge, die ausschließlich und gänzlich in einer Fremdsprache angeboten werden, sind in Italien seitdem verfassungswidrig. Insbesondere dem Unterrichtsministerium, das der Berufung des Politecnico beigetreten war, schrieb der Oberste Verwaltungsgerichtshof (Consiglio di Stato) ins Stammbuch, die >Internationalisierung der Hochschulen dürfe die italienische Sprache nicht zugunsten des Englischen in eine marginale Position abdrängen, auch nicht in einzelnen Fächern.

In Deutschland werden laut Auskunft der HRK/Hochschulbarometer von 18.397 staatlich anerkannten Studiengängen bereits 1.575 als international , d.h. ausschließlich auf Englisch angeboten. ${ }^{35} \mathrm{Ihr}$ Anteil steigt seit Jahren. ${ }^{36}$ Aus reinem Opportunismus wird sogar Etikettenschwindel mit oft nur mäßig verständlichen, doch englisch klingenden Titeln getrieben, wenn diese für dennoch deutschsprachige und immer kleinteiligere Curricula werben sollen. ${ }^{37}$ Sehr oft existiert selbst für Angebote mit eindeutig regionalem oder Anwendungsbezug keine deutschsprachige Alternative.

Die TU München will 2020 sogar fast ihr gesamtes Lehrangebot in den weiterführenden Studiengängen auf Englisch umstellen. Am 30. September 2019 stellte es der bayrische Staatsminister für Wissenschaft und Kunst den Hochschulleitungen Bayerns sogar frei, im Widerspruch zum eigenen Hochschulgesetz auch alle grundständigen Studiengänge komplett auf Englisch umzustellen und entgegen bisher verpflichtender Praxis keine Zwillingsstudiengänge auf Deutsch mehr vorzuhalten. ${ }^{38}$

$\mathrm{Zu}$ allem Überfluss unterstützt nun sogar der DAAD (Deutscher Akademischer Austauschdienst), der im Ausland studienvorbereitende Deutschkurse anbietet, die Werbung einzelner Hochschulen für englischsprachige Studiengänge in Deutschland. ${ }^{39}$ All dies benachteiligt nicht nur deutschsprachige Studenten und Hochschullehrer, sondern bedroht auch die Qualität der Lehre und behindert die berufliche und gesellschaftliche Integration

Siehe Hochschulkompass.

Mocikat: Die Sprache in den Naturwissenschaften.

Vitzthum: Fachidioten erobern die deutschen Universitäten.

Nähere Ausführungen hierzu unter der Rubrik »Sprachenpolitik an Hochschulen « auf den Internetseiten des Arbeitskreises Deutsch als Wissenschaftssprache (<http://adawis.de/hochschullehre/sprachenpolitik-an-hochschulen $>$ ).

9 Vgl. <https://daad-publish.reflact.com/content/connect/c1/7/en/events/event/shared/3332715/ event_landing.html?sco-id=8793472> (Zugriff: 7.11.2019). 
ausländischer Absolventen. Genau deshalb verfügte die dänische Regierung vor kurzem die Reduktion englischsprachiger Studienprogramme.

Fazit: Hinsichtlich des Schutzes der Grundrechte, insbesondere der Wissenschaftsfreiheit und der Freiheit der Berufswahl, gleichen sich die Verfassungen Italiens und Deutschlands. Ein Verfassungsstreit zur Sprachlichkeit der akademischen Lehre würde in Deutschland daher wohl ähnlich klar gegen >English only< ausfallen wie in Italien..$^{40}$ Der Arbeitskreis Deutsch als Wissenschaftssprache e. V. (www.adawis.de), dem der Autor als Mitgründer und Vorstandsmitglied seit 2007 angehört, würde jeden Hochschulangehörigen, der oder die sich gegen ein Englisch-Diktat in der Lehre zur Wehr setzt, im Rahmen seiner Möglichkeiten unterstützen.

\subsection{Tutzinger Forderungen zur Sprache der Lehre an deutschen Hochschulen}

Mit den aus `English only` resultierenden bildungspolitischen Fragen beschäftigte sich die Tagung »Die Sprache von Forschung und Lehre: Lenkung durch Konzepte der Ökonomie? « im Februar 2018 in Tutzing am Starnberger See. Der Arbeitskreis Deutsch als Wissenschaftssprache e. V., die Akademie für Politische Bildung in Tutzing und das Zentrum für Europäische Bildung in Zagreb hatten sie gemeinsam ausgerichtet. ${ }^{41}$

Alle Teilnehmer betonten, dass gerade die Wissenschaft vom mehrsprachigen interkulturellen Austausch profitiert. Viele beklagten, dass `English only< diesen Austausch verflacht, die Vielfalt der Erkenntnis mindert sowie die Freiheit der Lehre und der Berufswahl einschränkt. Die Wahl der Sprache(n) für die akademische Lehre sei deshalb nicht nur in Italien keine Frage der hochschulpolitischen und ökonomischen Zweckmäßigkeit, sondern habe grundsätzlich Verfassungsrang - auch in anderen Ländern, z.B. in Deutschland. Zahlreiche Teilnehmer, namhafte Wissenschaftler und Verwaltungsfachleute unterzeichneten deshalb die Tutzinger Forderungen zur Sprache der Lehre an deutschen Hochschulen:

1. Alle Studiengänge sind in deutscher Sprache anzubieten.

2. Studiengänge für Master-Abschlüsse können zusätzlich zum deutschen Studiengang auch (modulweise oder vollständig) in einer anderen Sprache angeboten werden.

40 Nähere Ausführungen hierzu auf den Internetseiten des Arbeitkreises Deutsch als Wissenschaftssprache, unter Hochschullehre > Rechtliche Aspekte: <http://adawis.de/hochschullehre/ rechtliche-aspekte> (Zugriff: 7.11.2019).

41 Tagungsband: Münch et al.: Die Sprache von Forschung und Lehre. 
3. Für das Studium von Fremdsprachen und nicht deutschsprachiger Kulturen sind fachspezifisch anderssprachliche Lösungen zulässig und erwünscht.

4. Für Studenten nichtdeutscher Muttersprache sind Deutschkurse (Allgemein- und Fachsprache) verbindlich vorzusehen und mit Leistungspunkten zu honorieren, so dass im Laufe des Studiums zunehmend deutschsprachige Lehrveranstaltungen belegt werden können.

5. In Promotionsprogrammen gelten die Grundsätze 2) bis 4) analog.

Die Unterzeichner sehen die Bundesländer in der Pflicht, ihre Hochschulgesetze entsprechend zu ergänzen. Notfalls müsse der Bund dafür eine Rahmenkompetenz schaffen.

\section{Ausblick}

Wissenschaft zu treiben heißt mehr, als nur international zu kommunizieren. Die Gleichsetzung Englisch = Wissenschaftssprache ist ein verhängnisvolles wissenschaftstheoretisches Missverständnis, das die Propositionen der Wissenschaft selbst in Frage stellt. Nach wie vor birgt die Mehrsprachigkeit Europas auch in den MINT-Fächern ein Erkenntnispotenzial, das es insbesondere in deren akademischer Lehre zu bewahren und wieder zu entdecken gilt. Der galoppierende Rückzug der deutschen Sprache sowie anderer Sprachen aus ganzen Wissens- und Gesellschaftsbereichen wird nicht nur die kulturelle Vielfalt des Globus, sondern allmählich auch das Erkenntnispotenzial der davon betroffenen Wissenschaftsfächer beschädigen.

Im Sinne ihrer nachhaltigen Inter-Nationalisierung müssen die Wissenschaftspolitik, die Universitäten und andere Forschungseinrichtungen Maßnahmen im Sinne einer aktiven Sprachenpolitik einleiten. Das Englische als internationales Verständigungsmedium auch unter Wissenschaftlern steht dabei nicht zur Disposition.

Es wird jedoch nötig sein,

- schon frühzeitig die rezeptive und aktive Mehrsprachigkeit zu fördern,

- den europäischen Landessprachen verstärkt die Rolle der inter- und transdisziplinären, auch hochschulinternen Verständigungssprachen zuzuweisen,

- die bereits existierenden Sprachkurse für Ausländer auszubauen, ihren Besuch außer bei Kurzaufenthalten verpflichtend zu machen und durch curriculare Leistungspunkte erfolgsentsprechend $\mathrm{zu}$ honorieren. 
Grundsätzlich sollten Wissenschaftler zumindest passiv mehrere Sprachen beherrschen, also nicht nur ihre Muttersprache und das Englische. Der Weg dorthin muss spätestens in den Mittelstufen der Gymnasien und weiterführenden Schulen beginnen.

\section{Literaturverzeichnis}

Arbeitskreis Deutsch als Wissenschaftssprache. Leitlinien ... weil Wissenschaft alle angeht. September 2019. <http://adawis.de/fileadmin/user_upload/Seiten/Mitglied_werden/ RZ_ADAWiS_Flyer_Oktober2019_web.pdf > .

Bhattacharjee, Yudhijit: Saudi Universities Offer Cash in Exchange for Academic Prestige. »Science« 334.6061 (2011), S. 1344-1345.

Dem demografischen Wandel entgegen. Wie schrumfende Hochschulstandorte internationale Studierende gewinnen und halten. Studie des SVR-Forschungsbereichs 2019-1. Bundesministerium für Bildung und Forschung 2019. <https://www.svr-migration. de/wp-content/uploads/2019/03/SVR_FB_Schrumpfende_Hochschulstandorte.pdf>

Dieter, Hermann H.; Adams, Martina: Praxis der Internationalisierung an den Öffentlichen Hochschulen - da läuft etwas falsch! <http://adawis.de/fileadmin/user_upload/Dieter_Adam_GWK_2017.pdf>

Drewer, Petra: Die kognitive Metapher als Werkzeug des Denkens. Zur Rolle der Analogie bei der Gewinnung und Vermittlung wissenschaftlicher Erkenntnisse. Tübingen: Gunter Narr Verlag 2003.

Finke, P. L. W.: Das Scheitern der Wissenschaft als Hoffnungsträgerin. Neue Rollen für die Profis, die Amateure und die Zivilgesellschaft. Vortrag, gehalten am 24.10.2019 im Deutschen Klimarechenzentrum Hamburg (DKRZ).

Fischer, Roland: Vermessene Wissenschaft. »Neue Zürcher Zeitung «(10.11.2011). <https:// www.nzz.ch/vermessene_wissenschaft-1.11851334> (Zugriff: 7.11.2019).

Heisenberg, Werner: Der Teil und das Ganze. Gespräche im Umkreis der Atomphysik. 2. Aufl. München: Piper 1973.

Hettiger, Andreas: Sprachenpolitik an deutschen Hochschulen. Grundlagen und Perspektiven. Baltmannsweiler: Schneider Verlag Hohengehren 2019.

Hochschulkompass. Ein Angebot der Hochschulrektorenkonferenz. <https://www.hochschulkompass.de/home.html> (Zugriff: 28.10.2019).

Hvistendahl, Mara: China's Publication Bazaar. »Science« 342.6162 (2013), S. 1035-1039.

Kluge, Friedrich: Ethymologisches Wörterbuch der deutschen Sprache. Berlin, New York: de Gruyter 1999.

Kommission sieht schwere Fehler bei Bluttest-Affäre. »Süddeutsche Zeitung« (16.7.2019). $<$ https://www.sueddeutsche.de/gesundheit/heidelberg-brustkrebs-bluttest-skandal-1.4527497> (Zugriff: 7.11.2019).

Mocikat, Ralph: Die Diktatur der Zitatenindices: Folgen für die Wissenskultur. »GAIA«18/2 (2009), S. 100-103. <http://adawis.de/fileadmin/user_upload/Seiten/Stellungnahmen/ Publikationen_des_ADAWIS/GAIA.pdf $>$.

Mocikat, Ralph: Fertigwissen in der Einheitssprache. Was hat die "Bologna-Reform « mit Wissenschaftssprache zu tun? »Forschung und Lehre» 17 (2010), S. 652-653. 
Mocikat, Ralph: Qualitätsbewertung in den Naturwissenschaften mithilfe quantitativer Parameter: Ein Paradox? »Denkströme. Journal der Sächsischen Akademie der Wissenschaften« 5 (2010), S.90-102.<http://www.denkstroeme.de/heft-5/s_90-102_mocikat> (Zugriff: 7.11.2019).

Mocikat, Ralph: Die Sprache in den Naturwissenschaften: Herausforderungen in Zeiten der Internationalisierung. In: Mehrsprachigkeit und Elitenbildung im europäischen Hochschulraum. Hgg. N. Colin, J. Umlauf. Heidelberg: Synchron 2015, S. 57-68.

Mocikat, Ralph: Der Wert der Mehrsprachigkeit in den Naturwissenschaften und der Medizin. In: Mehrsprachigkeit im Wissenschaftsdiskurs. Ein Panorama der Möglichkeiten und Schwierigkeiten. Hgg. H. W. Giesen, A. Krause, P. Oster-Stierle, A. Raasch. NOMOS, Baden-Baden 2018, S. 269-282.

Mocikat, Ralph: Die Anglophonisierung der Wissenschaften als Folge ihrer Ökonomisierung? In: Die Sprache von Forschung und Lehre. Lenkung durch Konzepte der Ökonomie? Hgg. U. Münch, R. Mocikat, S. Gehrmann, J. Siegmund. Baden-Baden: Nomos (im Druck).

Münch, Richard: Wissenschaft im Wettbewerb strategisch operierender Universitäten. In: Die Sprache von Forschung und Lehre. Lenkung durch Konzepte der Ökonomie? Hgg. U. Münch, R. Mocikat, S. Gehrmann, J. Siegmund. Baden-Baden: Nomos (im Druck).

Münch, Ursula; Mocikat, Ralph; Gehrmann, Siegfried; Siegmund, Jörg (Hgg.): Die Sprache von Forschung und Lehre. Lenkung durch Konzepte der Ökonomie? Baden-Baden: Nomos (im Druck).

Oswalt, Philipp; Adams, Martina: Ohne Worte. Universitäten wollen um jeden Preis internationaler werden. Leider sprechen die ausländischen Studenten kaum Deutsch. So geht es nicht weiter. »Die Zeit« Nr. 13 (23.3.2017). <http://www.zeit.de/2017/13/ internationale-studenten-deutschkenntnisse-universitaet/komplettansicht?print> (Zugriff 7.11.2019).

Plickert, Philip: Wer sich nicht erpressen lässt, wird nicht gedruckt. Wie Zeitschriften ihre Bedeutung aufblasen und Wissenschaftler sich in Rankings nach oben mogeln. "Frankfurter Allgemeine Zeitung «, 7. 3.2012, S. 0N5 (Forschung und Lehre). <http://adawis. de/fileadmin/user_upload/FAZ_zu_Impactfaktor_7.3.2012.pdf $>$.

Saibil, Helen: Chaperone machines for protein folding, unfolding and disaggregation. »Nature Reviews Molecular Cell Biology« 14 (2013), S. 630-642.

Strategie der Wissenschaftsminister/innen von Bund und Ländern für die Internationalisierung der Hochschulen in Deutschland (Beschluss der 18. Sitzung der Gemeinsamen Wissenschaftskonferenz am 12. April 2013 in Berlin). <https://www.bmbf.de/files/ aaaInternationalisierungsstrategie_GWK-Beschluss_12_04_13.pdf $>$.

Thielmann, Winfried: Deutsche und englische Wissenschaftssprache im Vergleich: Hinführen - Verknüpfen - Benennen. Heidelberg: Synchron 2009.

Trabant, Jürgen: Globalesisch für europäische Mehrsprachigkeit und Sprachengerechtigkeit? In: Mehrsprachigkeit und Elitenbildung im europäischen Hochschulraum. Hgg. N. Colin, J. Umlauf. Heidelberg: Synchron 2015, S. 57-68.

Trabant, Jürgen: Weltansichten. Wilhelm von Humboldts Sprachprojekt. München: Beck 2012.

Tutzinger Forderungen zur Sprache der Lehre an deutschen Hochschulen. <http://adawis. de/fileadmin/user_upload/Tutzinger_Forderungen_2018_01.pdf $>$.

Vitzthum, Thomas: Fachidioten erobern die deutschen Universitäten. »Die Welt«, 27.9.2015. $<$ https://www.welt.de/politik/deutschland/article146904583/Fachidioten-eroberndie-deutschen-Universitaeten.html> (Zugriff: 7.11.2019).

Weizsäcker, Carl Friedrich von: Die Einheit der Natur. München: Carl Hanser Verlag 1971. 